\title{
Chorioamnionitis and Neonates; Which Strategy?
}

\author{
Seyyed-Abolfazl Afjeh, $\mathrm{MD}^{1,2^{*}}$; Mohammad Kazem Sabzehei, $\mathrm{MD}^{3}$; Roxana Mansor Ghanaie, $\mathrm{MD}^{2}$; Mahdiyeh Karimizadeh, $\mathrm{MD}^{4}$; Ahmad \\ Reza Shamshiri, MD, PhD ${ }^{5}$; Fatemeh Esmaili, BSc $^{1}$ \\ 'Mahdieh Medical Center, Shahid Beheshti University of Medical Sciences, Tehran, Iran \\ ${ }^{2}$ Pediatrics Infections Research Center, Shahid Beheshti University of Medical Sciences, Tehran, Iran \\ ${ }^{3}$ Hamadan University of Medical Sciences, Hamadan, Iran \\ ${ }^{4}$ Mofid Children Hospital, Shahid Beheshti University of Medical Sciences, Tehran, Iran \\ ${ }^{5}$ Department of Epidemiology and Biostatistics, School of Public Health, Tehran University of Medical Sciences, Tehran, Iran
}

\begin{abstract}
Background: Chorioamnionitis (CAM) is one of the major risk factors for neonatal early-onset sepsis (EOS). Different international guidelines have been developed for diagnosis and care of such neonates. This research aimed to evaluate our neonates and compare them with the guidelines.

Methods: This prospective cohort study was conducted during five years (March 2012 to March 2017), and comprised of neonates (any gestational age) born to mothers with CAM (any criteria). The neonates' clinical findings and interventions were collected and analyzed.

Results: In total, out of 28,988 live born neonates, CAM was found in mothers of 169 neonates (1.7\%). Among the studied neonates, $30.8 \%$ were born $\leq 34$ week of gestation, $39 \%$ had birth weight $<2500 \mathrm{~g}$, and $58.6 \%$ were asymptomatic. Out of 99 asymptomatic neonates, 47 were observed near mothers and 52 admitted to the neonatal intensive care unit (NICU). The frequency of abnormal tests was $23.07 \%$ in asymptomatic vs. $35.7 \%$ in symptomatic neonates; three neonates developed culture positive EOS $(2.75 \%)$ and $68.05 \%$ of the neonates received antibiotics. The length of stay was $2.59 \pm 1.13$ (median $=2.00, I Q R=$ $1.00)$ days in asymptomatic vs. $15.15 \pm 13.67$ (median $=7.00, \mathrm{IQR}=15.25)$ days in symptomatic neonates $(P<0.001)$.

Conclusion: The use of guidelines increased the length of stay, lab tests, and antibiotics in asymptomatic and neonates with negative blood culture. In addition to the mother-neonate separation, these guidelines may increase nosocomial infection, antibiotic resistance, and costs; therefore, new guidelines are needed to be developed.

Keywords: Chorioamnionitis, Neonatal sepsis, Newborn, Prematurity

Cite this article as: Afjeh SA, Sabzehei MK, Mansor Ghanaie R, Karimizadeh M, Shamshiri AR, Esmaili F. Chorioamnionitis and neonates; which strategy? Arch Iran Med. 2020;23(7):480-487. doi: 10.34172/aim.2020.45.
\end{abstract}

Received: July 20, 2019, Accepted: February 23, 2020, ePublished: July 1, 2020

\section{Introduction}

Chorioamnionitis (CAM) is a common pregnancy complication manifested by inflammation or infection or both of the chorion or amnion, ${ }^{1}$ and has been reported since 1961. ${ }^{2}$ The prevalence of CAM varies based on clinical, microbiological, histological diagnostic criteria from $0.5-10 \%$ in normal pregnancies to $25 \%$ in preterm pregnancies. ${ }^{3,4}$

CAM can increase the risk of serious maternal and fetal infection and its outcome, such that the prevalence of EOS in the late-preterm (LPT) and full-term (FT) neonates born to mothers with CAM was reported $1-3 \%$ in those receiving intra-partum antibiotic prophylaxis (IAP) and 5-8\% without therapy., ${ }^{5,6}$

Diagnosis is the main challenge in CAM. Given the limited facilities in the majority of centers, microbiological (positive culture of amniotic fluid or chorioamnion) and histopathologic (pathologic examination of placenta) tests cannot be performed and only clinical methods are used. ${ }^{7}$ Some studies have introduced maternal fever (MF), which can have infectious or non-infectious causes, ${ }^{1,8}$ and some have used the criteria of Gibbs et al including: intrapartum fever (IPF), with at least two of the following signs: increased maternal heart rate ( $>100$ per min), fetal heart rate $(>160$ per min), maternal white blood cells $(>15000$ cell $/ \mathrm{mm}^{3}$ ), painful uterus, and foul-smelling amniotic fluid. ${ }^{9}$ Grunberg et al reported that CAM is diagnosed based on MF and one other sign by $68.5 \%$ of gynecologists, based on only MF by $26.4 \%$ of gynecologists, and based on other symptoms by $4.7 \%$ of gynecologists. ${ }^{10}$ However, CAM is definitively diagnosed only through histological chorioamnionitis (HCA) assessment, and clinical and microbiological signs have low positive predictive value.

In the next stage, neonatal management is the main challenge. The Center for Disease Control and the Committee on Fetus and Newborn recommended lab tests and antibiotic (AB) therapy for all neonates born to mothers with CAM, ${ }^{11,12}$ which faced with widespread oppositions specifically for asymptomatic LPT and FT neonates. ${ }^{13-16}$ The American College of Obstetrician and 
Gynecologists recommended using the term intrauterine inflammation or infection or both (Triple I) instead of $\mathrm{CAM}^{1}$ and the American Academy of Pediatrics developed guidelines for neonates born at $\leq 346 / 7$ weeks and $\geq 35$ 0/7 weeks of gestational age with EOS to make more accurate decisions for maternal CAM diagnosis, neonatal care for hospitalization, conduction of lab test and administration of antibiotics. ${ }^{17}$

The present study aimed to evaluate the provided guidelines and our management of neonates born to mothers with CAM in our center, and also compare the results with other studies.

\section{Materials and Methods}

This prospective cohort study was conducted in Mahdiyeh Medical Center, affiliated to the Shahid Beheshti University of Medical Sciences (SBUMS), between March 2012 and March 2017 after obtaining approval from the Ethics Committee and informed consent. All neonates (regardless of gestational age) born to mothers with CAM (regardless of criteria) were included in the study. A Sample size of 174 was calculated using Cohen's formula with 0.05 type one error, $80 \%$ statistical power and 0.3 effect size (which can test about 20\% differences between groups with maximum variance).

In this study, the diagnosis of CAM was based on: clinical suspicion of the gynecologist, IPF $\left(\mathrm{T}>37.8^{\circ} \mathrm{C}\right)$, maternal leukocytosis (WBC >15000), maternal tachycardia (HR $>100 / \mathrm{min}$ ), fetal tachycardia (FHR $>160 / \mathrm{min}$ ), uterus tenderness, vaginal secretion or foul-smelling amniotic fluid (the Triple I categorization had not been developed yet in the early years of this study).

The demographic information of the mothers included gestational age, premature rupture of membranes (PROM), type of delivery, IAP, and IPF. The demographic information of the neonates included gestational age, birth weight, sex, presence or absence of clinical symptoms, performed tests (CBC, CRP, B/C, and $\mathrm{LP}$ ), type and duration of antibiotic therapy, length of stay, and neonatal outcome.

All symptomatic neonates or neonates with EOS signs and symptoms $\left(\mathrm{T} \geq 38^{\circ} \mathrm{C}\right.$ or $\mathrm{T}<35^{\circ} \mathrm{C}$, hypotension, RDS, cyanosis, apnea, acidosis, lethargy or irritable, abdominal distention or bilious vomiting, petechial, bleeding, etc.) ${ }^{3}$ were admitted to the NICU and received antibiotics after performing the tests. The asymptomatic neonates were observed for 6-12 hours in the NICU, then transferred to the mother's room if they were asymptomatic and feeding tolerance occurred; if the neonates remained asymptomatic for 24-48 hours, they were discharged with their mothers. Seven asymptomatic neonates received only laboratory tests and antibiotics were not administered, whereas 45 asymptomatic neonates (based on CAM criteria) were admitted. The diagnosis of EOS was based on blood or cerebrospinal fluid (CSF) culture in the first three days after the birth, along with receiving antibiotics for more than five days, or death before the 5 th day after birth while antibiotics were administered. ${ }^{3}$

The length of primary antibiotic therapy was 48 hours (until negative blood culture result); however, antibiotics continued based on the neonatologist's order in case of the presence of clinical symptoms or abnormal test results.

\section{Statistical Methods}

Categorical variables were reported as count and percentage, and quantitative variables as mean with standard deviation (SD) or median with interquartile range (IQR). For data analysis, we used chi-square (or Fisher's exact test if appropriate) and independent sample $t$ test or MannWhitney U test to compare the frequency of categorical and quantitative variables between study groups (symptomatic vs. asymptomatic neonates), respectively. $P$ values less than 0.05 were considered statistically significant.

\section{Results}

A total of 28988 live births occurred in our center during the study period, out of which 169 neonates (1.7\%) were born to mothers with CAM. The mean gestational age was $35.37 \pm 4.04$ weeks and the mean birth weight was $2619.35 \pm 868.1 \mathrm{~g}$. In addition, 52 neonates $(30.8 \%)$ were born $\leq 34$ th week of gestation and $66(39 \%)$ neonates were born with a birth weight of $\leq 2500 \mathrm{~g}$ (Table 1). According to the diagnosis of maternal CAM based on clinical findings, 107 (63\%) mothers had IPF (Table 2).

Although this center did not provide Group B Strep (GBS) screening, all mothers with CAM received IAP including ampicillin $(\mathrm{n}=159)$, gentamicin $(\mathrm{n}=152)$, and clindamycin $(\mathrm{n}=108)$.

Out of 169 neonates under investigation, 99 neonates (58.6\%) were asymptomatic ( 92 neonates were born $>34$ th week of gestation), of whom 47 neonates were observed near mothers, and 7 neonates were hospitalized without antibiotic therapy. Therefore, only 45 asymptomatic and 70 symptomatic neonates were admitted to the NICU and received antibiotics (Tables 1 and 2).

For the 122 admitted neonates, CBC $(\mathrm{n}=122)$, CRP $(\mathrm{n}=122), \mathrm{B} / \mathrm{C}(\mathrm{n}=109)$ and LP $(\mathrm{n}=10)$ were performed and were positive in $9 \%, 18 \%, 2.7 \%$, and $10 \%$ of cases, respectively, specifically in symptomatic neonates (Tables 1 and 2).

Antibiotics were administered to 115 neonates (68\%), of whom $45.45 \%$ were asymptomatic. The maternal CAM criteria in these neonates included: No MF ( $\mathrm{n}=41 / 62$; 66.1\%), Only MF ( $\mathrm{n}=13 / 19 ; 68.4 \%), M F+1$ Sign ( $=$ $22 / 37 ; 59.4 \%), M F+2$ Signs $(n=39 / 51 ; 76.5 \%)$, and STI ( $\mathrm{n}=40 / 53 ; 75.5 \%)$ (Tables 1 and 2 ).

The administered antibiotics were ampicillin $(\mathrm{n}=111)$, gentamicin $(n=110)$, and amikacin $(n=30)$. The duration of antibiotic therapy was $<3$ days in 58 neonates, 4-7 days in 34 neonates, 8-14 days in 11 neonates, and $>14$ days in 
Table 1. Basic Characteristics of Neonates with Maternal CAM

\begin{tabular}{|c|c|c|c|c|}
\hline Characteristic & Total $(n=169)$ & Asymptomatic $(\mathrm{n}=99)$ & Symptomatic $(\mathrm{n}=70)$ & $P$ value \\
\hline \multicolumn{5}{|l|}{ Sex } \\
\hline Male & $85(50.30 \%)$ & $48(48.98 \%)$ & $37(52.85 \%)$ & \multirow[t]{2}{*}{$0.58+$} \\
\hline Female & $84(49.70 \%)$ & $51(51.02 \%)$ & $33(47.15 \%)$ & \\
\hline \multicolumn{5}{|l|}{ Birth weight(g) } \\
\hline$\leq 1500$ & $24(14.20 \%)$ & $0(0.00 \%)$ & $24(34.29 \%)$ & \multirow{3}{*}{$<0.001 \dagger$} \\
\hline $1501-2500$ & $42(24.85 \%)$ & $13(13.13 \%)$ & $29(41.43 \%)$ & \\
\hline$>2500$ & $103(60.95 \%)$ & $86(86.87 \%)$ & $17(24.29 \%)$ & \\
\hline \multicolumn{5}{|l|}{ Gestational age (wk) } \\
\hline$(>34)$ & $117(69.23 \%)$ & $92(92.93 \%)$ & $25(35.71 \%)$ & \multirow[t]{2}{*}{$<0.001 t$} \\
\hline$(\leq 34)$ & $52(30.77 \%)$ & $7(7.07 \%)$ & $45(64.29 \%)$ & \\
\hline \multicolumn{5}{|l|}{ WBC } \\
\hline Abnormal (>24000) & $11(9.02 \%)$ & $7(13.46 \%)$ & $4(5.71 \%)$ & \multirow[t]{2}{*}{$0.20+$} \\
\hline Normal & $111(90.98 \%)$ & $45(86.54 \%)$ & $66(94.29 \%)$ & \\
\hline \multicolumn{5}{|l|}{ CRP } \\
\hline Positive & $22(18.03 \%)$ & $5(9.62 \%)$ & $17(24.29 \%)$ & \multirow[t]{2}{*}{$0.04+$} \\
\hline Negative & $100(81.97 \%)$ & $47(90.38 \%)$ & $53(75.71 \%)$ & \\
\hline \multicolumn{5}{|l|}{$\mathrm{BC}$} \\
\hline Positive & $3(2.75 \%)$ & $0(0 \%)$ & $3(4.55 \%)$ & \multirow[t]{2}{*}{$0.28+$} \\
\hline Negative & $106(97.25 \%)$ & $43(100 \%)$ & $63(95.45 \%)$ & \\
\hline \multicolumn{5}{|l|}{ LP } \\
\hline Abnormal & $1(10.00 \%)$ & $0(0.00 \%)$ & $1(20.00 \%)$ & \multirow[t]{2}{*}{$1.00+$} \\
\hline Normal & $9(90.00 \%)$ & $5(100 \%)$ & $4(80.00 \%)$ & \\
\hline \multicolumn{5}{|l|}{ Antibiotic in neonate } \\
\hline Yes & $115(68.05 \%)$ & $45(45.45 \%)$ & $70(100 \%)$ & \multirow[t]{2}{*}{$<0.001+$} \\
\hline No & $54(31.95 \%)$ & $54(54.55 \%)$ & $0(0.00 \%)$ & \\
\hline \multicolumn{5}{|l|}{ Chorioamnionitis (CAM) } \\
\hline No maternal fever & $62(36.69 \%)$ & $30(30.30 \%)$ & $32(45.71 \%)$ & \multirow{4}{*}{$0.22+$} \\
\hline Maternal fever only & $19(11.24 \%)$ & $13(13.13 \%)$ & $6(8.57 \%)$ & \\
\hline Maternal fever +1 sign & $37(21.89 \%)$ & $24(24.24 \%)$ & $13(18.57 \%)$ & \\
\hline Maternal fever +2 signs & $51(30.18 \%)$ & $32(32.32 \%)$ & $19(27.14 \%)$ & \\
\hline \multicolumn{5}{|l|}{ Suspected Triple 1} \\
\hline No & $116(68.64 \%)$ & $66(56.90 \%)$ & $50(43.10 \%)$ & \multirow[t]{2}{*}{$0.51+$} \\
\hline Yes & $53(31.36 \%)$ & $33(33.33 \%)$ & $20(28.57 \%)$ & \\
\hline Hospital course (days) & $\begin{array}{c}7.18 \pm 11.18 \\
(\text { median }=3.00, \mathrm{IQR}=4.00)\end{array}$ & $\begin{array}{c}2.59 \pm 1.13 \\
(\text { median }=2.00, \mathrm{IQR}=1.00)\end{array}$ & $\begin{array}{c}13.67 \pm 15.15 \\
(\text { median }=7.00, \mathrm{IQR}=15.25)\end{array}$ & $<0.001 \neq$ \\
\hline \multicolumn{5}{|l|}{ Outcome } \\
\hline Survived & $162(95.86 \%)$ & $99(100 \%)$ & $63(90.00 \%)$ & \multirow[t]{2}{*}{$0.002+$} \\
\hline Expired & $7(4.14 \%)$ & $0(0.00 \%)$ & $7(10.00 \%)$ & \\
\hline
\end{tabular}

CAM, chorioamnionitis; WBC, white blood cell; CRP, C-reactive protein; BC, blood culture; LP, lumbar puncture.

${ }^{+} P$ values are calculated using chi-square (or Fisher exact test if appropriate).

${ }^{\ddagger} P$ value is calculated using Mann-Whitney $U$ test.

12 neonates. Results from statistical analysis showed that mean gestational age of $\leq 34$ weeks and lower birth weight $(P<0.001)$ were among risk factors causing symptoms and necessitating antibiotic therapy (Table 2 ).

The length of stay in asymptomatic and symptomatic neonates was $2.59 \pm 1.13$ vs. $15.15 \pm 13.67$ days, respectively $\quad(P<0.001)$.

In total, seven symptomatic neonates ( $\leq 34$ weeks) with birth weights of $\leq 1000 \mathrm{~g}(\mathrm{n}=3), 1001-1500 \mathrm{~g}(\mathrm{n}=2)$, and 1501-2500 $\mathrm{g}(\mathrm{n}=2)$, and positive blood culture $(\mathrm{n}=$ 1) died because of Culture Positive EOS $(n=1)$, RDS ( $n=$ $5)$, and congenital anomaly $(\mathrm{n}=1)$.

\section{Discussion}

This prospective cohort study was conducted in Mahdiyeh Medical Center to compare mothers with CAM and their neonates based on CDC, COFN, ACOG, and AAP guidelines. In this study, the mean prevalence of CAM was $1.7 \%$, which is in the acceptable range of 0.5 to $10 \%$; whereas, CAM prevalence was $4 \%$ in Braun's study, 6.5\% in Jan's study, and 2-4\% FT and 25\% PT in Edmir's study. ${ }^{3-6}$

Although the prevalence of CAM increases with reduced gestational age $(87.3 \%<30$ weeks; and $47.3 \%<32$ weeks), its prevalence varies in different studies based on the 


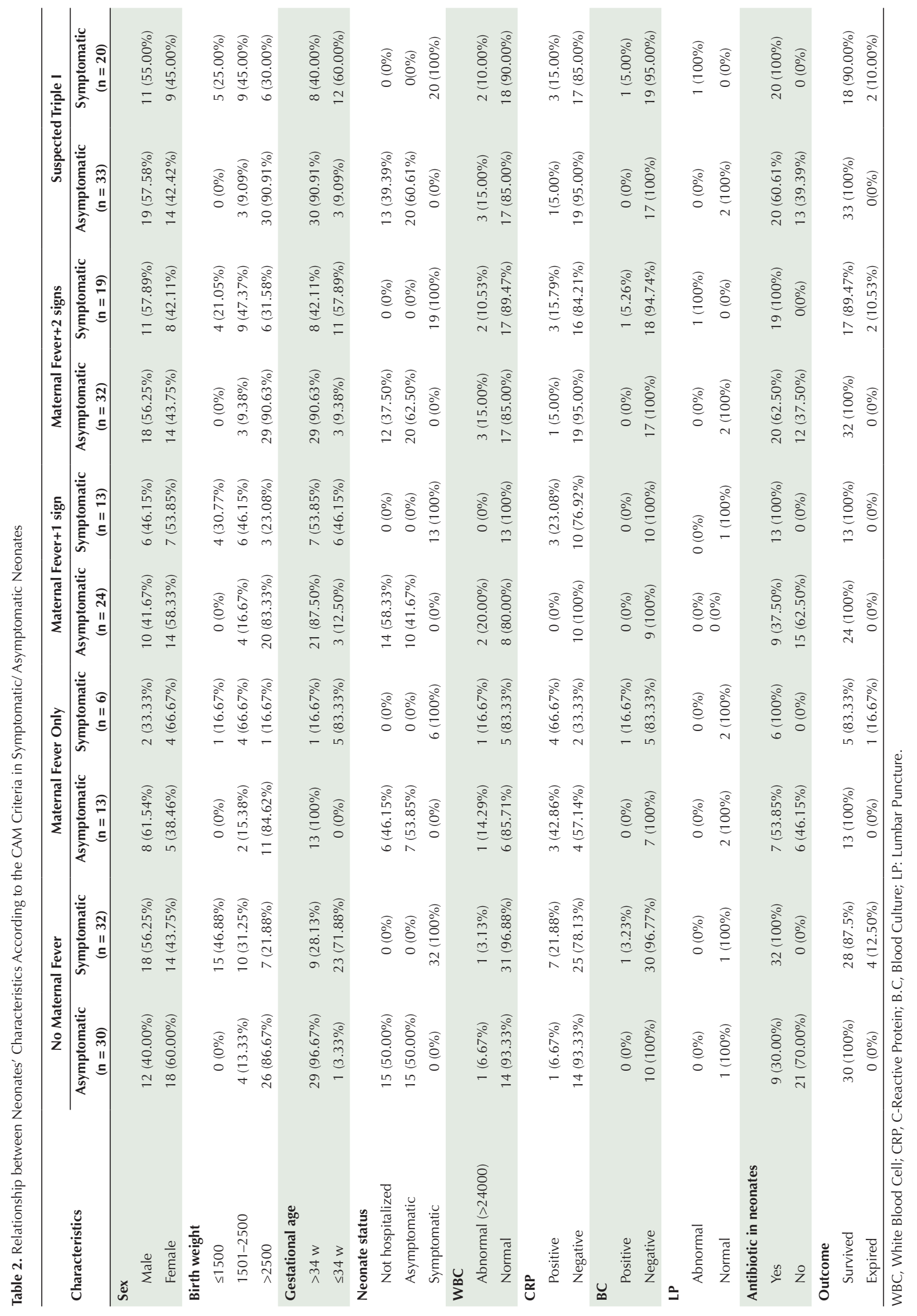


diagnostic criteria. Although definite diagnosis is based on the $\mathrm{HCA},{ }^{7}$ it is not widely used as it causes delay in diagnosis and treatment (approximately 48 hours after the delivery). Therefore, the majority of centers use clinical symptoms. Recently, ACOG proposed the term Triple I, based on which the mothers are divided into three groups of: only IPF, Suspected Triple I (STI), and confirmed Triple I. ${ }^{1,2}$ However, clinical symptoms are not definite criteria for CAM diagnosis.

In the present study, 62 mothers $(36.7 \%)$ were without IPF: maternal tachycardia $(\mathrm{n}=13), \mathrm{FHR}>160 / \mathrm{min}$ ( $\mathrm{n}$ $=2$ ), foul-smelling amniotic fluid $(\mathrm{n}=1)$ and clinical suspicion $(\mathrm{n}=46)$. In addition, 24 neonates were born prior to the 34 th week of gestation, 32 neonates were symptomatic, and one neonate's blood culture was positive for E. coli. This group can be regarded as CAM diagnosed by diagnostic criteria other than fever, accounting for $4.7 \%$ of all CAM cases. ${ }^{10}$

On the other hand, 19 mothers had only IPF, 37 mothers had IPF and one sign, 51 mothers had IPF and two signs, and 53 mothers were in the STI group. ${ }^{1}$ Totally, 107 mothers had IPF (63.3\%) in the present study vs. $81.02 \%$ in the study by Randis. ${ }^{18}$ Jan and Azuma reported IPF as a risk factor necessitating NICU admission for the neonates. ${ }^{6,19}$

The HCA studies have shown that the sensitivity of IPF is $42 \%$, which could increase to $60 \%$ if accompanied by other clinical symptoms. ${ }^{20,21}$ In Ericson's study, IPF was not observed in $92 \%$ of the HCA cases; whereas mothers with IPF had $59 \%$ of the HCA findings. ${ }^{22}$ Roman showed that only $33 \%$ of acute clinical chorioamnionitis (ACCA) cases had confirmed TI and $29.2 \%$ of their neonates had clinical symptoms of sepsis. ${ }^{23}$

All 169 mothers in the present study diagnosed with ACCA received IAP. Administration of IAP was recommended for Early Onset Group B. Streptococcus Infection (EOGBSI) prevention since 1980 and increased to $30 \%$ of pregnant women until 2010. ${ }^{24}$ Although the reduction of EOGBSI by $86 \%$ in 1990-2010 is attributed to this strategy, ${ }^{25}$ it causes short and longterm complications for mothers and neonates (microbial resistance, change in mother-to-infant microbial balance, and drug allergy). ${ }^{26}$ The problem is that many doctors, similar to our colleagues in the present study, prescribe IAP based on ACCA criteria (regardless of the maternal GBS status). ${ }^{27}$

CAM is regarded as an important risk factor for the incidence of premature birth, CP, PVL, IVH, EOS, seizure, pneumonia, BPD, and RDS in neonates. ${ }^{22}$ As a result, many studies have focused on the relationship between CAM and EOS in neonatal care. ${ }^{3,5,6,28-32}$

We considered the development course of guidelines proposed between 2012 and 2016 and tried to evaluate their outcomes.

According to $\mathrm{CDC}^{11}$ and $\mathrm{COFN}^{12}$ guidelines, all
169 neonates should be hospitalized, lab tests should be performed, and antibiotics should be administered; however, we hospitalized only 122 neonates (the tests were performed in only 7 neonates) and 115 neonates $(68 \%)$ received antibiotic therapy, among whom 70 neonates $(59.8 \%)$ were born 234 th week of gestation ( 45 asymptomatic neonates), and 45 symptomatic neonates $<34$ th week of gestation.

According to the ACOG guideline, out of 19 mothers with only IPF ( 5 neonates born $<34$ th week of gestation and 1 neonate born $\geq 34$ th week of gestation, all symptomatic), $6 / 19$ vs. $13 / 19$ should have been hospitalized and treated. Also, from 53 mothers with STI $(15$ neonates born $<34$ th week of gestation and 8 neonates born $\geq 34$ th week of gestation and symptomatic), $23 / 53$ vs. $40 / 53$ should have been hospitalized and treated, yielding a total of 29/72 vs. 53/72 who should have been hospitalized and treated, which indicates a reduction by approximately $50 \%$.

In general, $59.8 \%$ of neonates $>34$ weeks and $86.5 \%$ of neonates $\leq 34$ weeks were treated. As a result, making a decision based on the gestational age and symptoms resulted in reduced need for hospitalization, medical tests, and antibiotic therapy. In our study, $45 / 52$ of preterm (PT) $<34$ weeks neonates (86.5\%), 21/32 of late preterm (LPT) neonates $(65.6 \%)$, and $44 / 85$ of full term (FT) neonates $(51.7 \%)$ were hospitalized and treated. Also, the need for hospitalization and antibiotic therapy can be reduced by physical examination of the asymptomatic LPT and FT neonates, ${ }^{33}$ or using the Sepsis Risk Calculator (SRC), ${ }^{34,35}$ and in PT neonates ( $\leq 34$ weeks) based on AAP guideline. ${ }^{17}$ However, the results depend on future clinical studies.

In addition to gestational age, symptomatic or asymptomatic neonates, the type and value of tests are important factors. ${ }^{1,11,12,17}$ According to the guidelines, CBC (WBC, IT, ANC, Platelet), CRP (single or serial), and blood culture (conventional, modern, aerobic, anaerobic) are recommended. Although the role of IAP and technical error ${ }^{17,35}$ can lead to false negative or positive blood culture results, ${ }^{17}$ the culture negative sepsis (CNS) continues to remain a "medical myth" ${ }^{34}$

Lumbar puncture (LP) and CSF culture (before antibiotic therapy) are recommended for: neonates at high-risk of EOS, positive blood culture ${ }^{17}$ and abnormal test results. $^{35}$

In this study, CBC and CRP were performed for 122 neonates, blood culture $(\mathrm{B} / \mathrm{C})$ for 109 neonates, and LP for 10 neonates. According to the results, WBC was $>24000$ in 11 neonates (4 symptomatic), CRP was positive in 22 neonates (17 symptomatic), blood culture was positive in three symptomatic neonates, and LP was positive in one neonate (35 weeks, symptomatic, with positive CBC, CRP, B/C). Many studies have evaluated the sensitivity and specificity of these tests, ${ }^{6,32,35}$ but recently the AAP recommends only $\mathrm{B} / \mathrm{C}$ and $\mathrm{CSF} / \mathrm{C}$ before antibiotic therapy. ${ }^{17}$ 
In three neonates with positive blood culture including two neonates $(2 / 45,4.4 \%)$ born $<34$ th week of gestation and one neonate $(1 / 64,1.6 \%)$ in the 35 th week, CAM in their mothers was diagnosed based on: No MF ( $\mathrm{n}=$ 1, GA:33 weeks, CRP: Negative, B/C: E. coli), Only MF ( $\mathrm{n}=1$, GA:33 weeks, CRP: Positive, B/C: Staphylococcus epidermidis), and $\mathrm{MF}+2$ signs ( $\mathrm{n}=1, \mathrm{GA}=35$ weeks, CRP: Positive, B/C and CSF/C: Acinetobacter). All three neonates were symptomatic at birth. The neonate with positive blood culture for $E$. coli died one day after birth. The prevalence of EOS in the present study was $4.4 \%$ in neonates born $<34$ th week of gestation, which was higher than Randis's $(0.7 \%)^{18}$ and Roman's studies $(0.2 \%){ }^{23}$ but was similar to Benitz's study ${ }^{16}$ with addition of the neonate born in the 35th week of gestation. As a result, although CAM is prevalent, EOS reduced specifically in the neonates $>34$ th week of gestation (only one neonate with positive culture in our study). However, Azuma showed that 10 out of 12 neonates with positive blood culture had normal test results and no clinical symptoms. ${ }^{19}$ Although $75 \%$ of neonates received IAP in less than 4 hours before delivery, Venkatesh showed that the duration of antibiotic therapy until the delivery (CAM duration) had no impact on the incidence of complications and EOS. ${ }^{36}$

According to $\mathrm{CDC}^{11}$ and $\mathrm{COFN},{ }^{12}$ neonatal management (after obtaining medical history, and performing clinical examination and tests) is continued with initiation of antibiotics for all neonates (regardless of gestational age and symptoms). $\mathrm{ACOG}^{37}$ recommends the administration of antibiotics only for symptomatic neonates or the neonates born $<34$ th weeks of gestation. ${ }^{1}$ The APP recommends antibiotics only for high-risk neonates $\leq 346 / 7$ weeks and $\geq 350 / 7 .{ }^{17}$ The duration of antibiotic therapy must be maximally 48 hours in case of negative blood culture results, ${ }^{11}$ normalization of CRP and $\mathrm{CBC}$ tests, ${ }^{12}$ and resolution of clinical symptoms. However, the AAP recommends therapy only up to a negative blood culture result. ${ }^{17}$

In this study, $68 \%$ of the neonates received antibiotics, of whom 92 neonates received it for less than 7 days $(50 \%$ for less than 3 days) and 23 neonates received it for more than one week. In addition, 45 neonates were asymptomatic and born $>34$ th weeks of gestation. In Azuma's study, 76\% of the neonates received antibiotic for more than 7 days. ${ }^{19}$ Fortunately, prolonged empirical antibiotic therapy has been avoided in our unit since $2011 .^{38}$

Administration of antibiotics to neonates leads to NICU admissions, mother-neonate separation, impaired breastfeeding, increased antibiotic resistance, increased hospital costs, complications (LOS, NEC), and death. ${ }^{17}$ Jan disapproved the use of antibiotic even for a short period. ${ }^{6}$ The APP recommended the adherence to Antibiotic Stewardship Program (ASP). ${ }^{39}$ However, it is very important to distinguish between asymptomatic neonates born to mothers with CAM and asymptomatic neonates with EOS. ${ }^{3}$

Another important factor is the length of hospital stay, which is related to the duration of antibiotic therapy. This is because long-term antibiotics for asymptomatic neonates with negative blood culture ${ }^{30}$ increases the length of stay due to not adhering to international guidelines in $40 \%$ of the centers. ${ }^{12}$

In the present study, the length of stay was $2.02 \pm 0.62$ days for asymptomatic neonates without antibiotic therapy, $3.21 \pm 1.23$ days for asymptomatic neonates with antibiotic therapy, and $15.15 \pm 13.67$ days for symptomatic neonates with antibiotic therapy. The comparison of the first and second groups indicates early discharge of asymptomatic neonates with normal test results.

Out of 169 neonates in our study, 7 neonates (4.1\%) died. All of these neonates were born $<34$ th week of gestation and had a birth weight of $<2500 \mathrm{~g}$. The diagnosis of CAM in their mothers was as follows: No MF $(n=4)$, only MF ( $\mathrm{n}=1), M F+2$ sings $(\mathrm{n}=2)$, and blood culture positive for $E$. $\operatorname{coli}(\mathrm{n}=1)$. Edmir reported that $12.3 \%$ of the neonates died and this rate was $10.6 \%$ in LBW neonates. ${ }^{4}$ Although the rate of neonatal death ( $>35$ weaks) in this study was similar to Azuma's study, ${ }^{19}$ Wortham reported that the prevalence of death among neonates with sepsis was $21 \% .^{3}$

The limitations of the present study are: 1) Conduction of the study in one center, although as a perinatal center, high-risk pregnancies and deliveries are admitted. 2) The limited number of neonates in this study, especially for those analyses with sparse data. So, the issue of sparse data bias must be borne in mind. ${ }^{40}$ (3) Changes in international guidelines during the five years of the study period. (4) Lack of access to modern blood culture media. ${ }^{17}$ (5) The small number of neonates with EOS $(\mathrm{n}=3)$ for statistical analysis. (6) Although no neonate was re-admitted for sepsis during the study, there was no follow-up for probable long-term complications of antibiotic therapy.

In conclusion, our study showed that similar to other studies against CDC and COFN, more than $50 \%$ of our asymptomatic neonates born $>34$ th week of gestation did not receive antibiotics, had a short hospital course, antibiotic day and low EOS. Our study revealed that at present, the ACOG guidelines are effective in reducing hospitalization of neonates born $\geq 34$ th week of gestation ${ }^{1}$ and the AAP guideline should be considered in clinical trials on neonates $<34$ 6/7 week of gestation. ${ }^{17}$ This combination of guidelines (ACOG \& AAP) may reduce hospitalization, laboratory tests, antibiotic prescription, and other complications.

\section{Authors' Contribution}

SAA: study concept and design. MKS, FE and RMG: acquisition of data. SAA and ARS: analysis and interpretation of data. MKS, MK and RMG: drafting of the manuscript. SAA: critical revision of the manuscript for important intellectual content. ARS and FE: statistical analysis. SAA, MKS, RMG and MK: administrative, technical, and material support. SAA: study supervision. All authors read and 
approved the final manuscript.

\section{Conflict of Interest Disclosures}

Authors have no conflict of interest to declare.

\section{Ethical Statement}

This study was approved by ethics committee of Shahid Beheshti University of Medical Sciences.

\section{Acknowledgements}

We thank the director and the staff of the Pediatric Infections Research Center (PIRC) at SBUMS for their help in planning this research, and also our colleagues in the neonatal ward of Mahdiyeh Medical Center for their all-out cooperation in implementing this project.

\section{References}

1. Higgins RD, Saade G, Polin RA, Grobman WA, Buhimschi IA, Watterberg $\mathrm{K}$, et al. Evaluation and management of women and newborns with a maternal diagnosis of chorioamnionitis: summary of a workshop. Obstet Gynecol. 2016;127(3):42636. doi: 10.1097/AOG.0000000000001246.

2. Blanc WA. Pathways of fetal and early neonatal infection. Viral placentitis, bacterial and fungal chorioamnionitis. J Pediatr 1961;59:473-96. doi: 10.1016/s0022-3476(61)80232-1.

3. Wortham JM, Hansen NI, Schrag SJ, Hale E, Van Meurs $\mathrm{K}$, Sánchez PJ, et al. Chorioamnionitis and cultureconfirmed, early-onset neonatal infections. Pediatrics. 2016;137(1):e20152323. doi: 10.1542/peds.2015-2323.

4. Erdemir G, Kultursay N, Calkavur S, Zekioğlu O, Koroglu OA, Cakmak B, et al. Histological chorioamnionitis: effects on premature delivery and neonatal prognosis. Pediatr Neonatol. 2013;54(4):267-74. doi: 10.1016/j.pedneo.2013.03.012.

5. Braun D, Bromberger P, Ho NJ, Getahun D. Low rate of perinatal sepsis in term infants of mothers with chorioamnionitis. Am J Perinatol. 2016;33(2):143-50. doi: 10.1055/s-0035-1560045.

6. Jan IA, Ramanathan R, Cayabyab RG. Chorioamnionitis and management of asymptomatic infants $\geq 35$ weeks without Empiric Antibiotics. Pediatrics. 2017;140(4):e20172212. doi: 10.1542/peds.2017-2212.

7. Chan GJ, Silverman M, Zaman M, Murillo-Chaves A, Mahmud $A$, et al. Prevalence and risk factors of chorioamnionitis in Dhaka, Bangladesh. J Perinatol. 2016;36(12):1039-44. doi: 10.1038/jp.2016.150.

8. Riley LE, Celi AC, Onderdonk AB, Roberts DJ, Johnson LC, Tsen LC, et al. Association of epidural-related fever and noninfectious inflammation in term labor. Obstet Gynecol. 2011;117(3):58895. doi: 10.1097/AOG.0b013e31820b0503.

9. Yoder PR, Gibbs RS, Blanco JD, Castaneda YS, St Clair PJ. A prospective, controlled study of maternal and perinatal outcome after intra-amniotic infection at term. Am J Obstet Gynecol. 1983;145(6):695-701. doi: 10.1016/00029378(83)90575-6.

10. Greenberg MB, Anderson BL, Schulkin J, Norton ME, Aziz N. A first look at chorioamnionitis management practice variation among US obstetricians. Infect Dis Obstet Gynecol. 2012;2012:628362. doi: 10.1155/2012/628362

11. Verani JR, McGee L, Schrag SJ. Prevention of perinatal group B streptococcal disease - Revised Guidelines From CDC, 2010. MMWR Recomm Rep. 2010;59(RR-10):1-36.

12. Polin RA. Committee on Fetus and Newborn. Management of neonates with suspected or proven early-onset bacterial sepsis. Pediatrics. 2012;129(5):1006-15. doi: 10.1542/ peds.2012-0541.

13. Van Herk W, el Helou S, Janota J, Hagmann C, Klingenberg C, Staub E, et al. Variation in current management of term and late-preterm neonates at risk for early-onset sepsis: an international survey and review of guideline. Pediatr Infect Dis J. 2016;35(5):494-500. doi: 10.1097/INF.0000000000001063.
14. Malloy $\mathrm{MH}$. Chorioamnionitis: epidemiology of newborn management and outcome United States 2008. J Perinatol. 2014;34(8):611-5. doi: 10.1038/jp.2014.81.

15. Shakib J, Buchi K, Smith E, Young PC. Management of newborns born to mothers with chorioamnionitis: is it time for a kinder, gentler approach? Acad Pediatr. 2015;15(3):340-4. doi: 10.1016/j.acap.2014.11.007.

16. Benitz WE, Wynn JL, Polin RA. Reappraisal of guidelines for management of neonates with suspected early-onset sepsis. J Pediatr. 2015;166(4):1070-4. doi: 10.1016/j. jpeds.2014.12.023

17. Puopolo KM, Benitz WE, Zaoutis TE. COMMITTEE ON FETUS AND NEWBORN, COMMITTEE ON INFECTIOUS DISEASES. Management of neonates born at $\geq 35$ 0/7 weeks' gestation with suspected or proven early-onset bacterial sepsis. Pediatrics. 2018;142(6):e20182894. doi: 10.1542/peds.2018-2896.

18. Randis TM, Rice MM, Myatt L, Tita ATN, Leveno KJ, Reddy UM, et al. Incidence of early-onset sepsis in infants born to women with clinical chorioamnionitis. J Perinat Med. 2018;46(8):92633. doi: 10.1515/jpm-2017-0192.

19. Azuma D, Boulais J. Does maternal chorioamnionitis have to equal antibiotics in an asymptomatic infant?. J Perinatol. 2018;38(7):778-80. doi: 10.1038/s41372-018-0141-8.

20. Curtin WM, Katzman PJ, Florescue H, Metlay LA. Accuracy of signs of clinical chorioamnionitis in the term parturient. J Perinatol. 2013;33(6):422-8. doi: 10.1038/jp.2012.135.

21. Tokumasu H, Hinotsu S, Kita F, Kawakami K. Predictive value of clinical chorioamnionitis in extremely premature infants. Pediatr Int. 2013;55(1):35-8. doi: 10.1111/ped.12036.

22. Ericson JE, Laughon MM. Chorioamnionitis: implications for the neonate. Clin Perinatol. 2015;42(1):155-ix. doi: 10.1016/j. clp.2014.10.011.

23. Roman A, Cruz Y, Sloane A, McElwee D, Carola D, Berghella $\mathrm{V}$, et al. Impact of new "Triple I" classification on clinical chorioamnionitis incidence and neonatal sepsis. Am J Obstet Gynecol. 2018;218(1):s504-5. doi: 10.1016/J. ajog.2017.11.382.

24. Schrag SJ, Farley MM, Petit S, Reingold A, Weston EJ, Pondo T, et al. Epidemiology of Invasive Early-Onset Neonatal Sepsis, 2005 to 2014. Pediatrics. 2016;138(6):e20162013. doi: 10.1542/peds.2016-2013.

25. Schrag SJ, Verani JR. Intrapartum antibiotic prophylaxis for the prevention of perinatal group B streptococcal disease: experience in the United States and implications for a potential group B streptococcal vaccine. Vaccine. 2013;31 Suppl 4:D20-D26. doi: 10.1016/j.vaccine.2012.11.056.

26. Braye K, Ferguson J, Davis D, Catling C, Monk A, Foureur M. Effectiveness of intrapartum antibiotic prophylaxis for early-onset group B Streptococcal infection: An integrative review. Women Birth. 2018;31(4):244-53. doi: 10.1016/j. wombi.2017.10.012.

27. The Royal Australian and New Zealand College of Obstetricians and Gynaecologists (RANZCOG). Maternal Group B Streptococcus in pregnancy: screening and management. 2016. Available from: https://ranzcog.edu.au/RANZCOG_ SITE/media/RANZCOG-MEDIA/Women $\% 27 \mathrm{~s} \% 20 \mathrm{Health}$ Statement\%20and\%20guidelines/Clinical-Obstetrics/ Maternal-Group-B-Streptococcus-in-pregnancy-screeningand-management-(C-Obs-19).pdf?ext=.pdf.

28. Avila C, Willins JL, Jackson M, Mathai J, Jabsky M, Kong A, et al. Usefulness of two clinical chorioamnionitis definitions in predicting neonatal infectious outcomes: a systematic review. Am J Perinatol. 2015;32(11):1001-9. doi: 10.1055/s-00351547325.

29. Kuzniewicz MW, Puopolo KM, Fischer A, Walsh EM, Li S, Newman TB, et al. A quantitative, risk based approach to the management of neonatal early-onset sepsis. JAMA Pediatr. 2017;171(4):365-71. doi: 10.1001/jamapediatrics.2016.4678. 
30. Money N, Newman J, Demissie S, Roth P, Blau J. Antimicrobial stewardship: antibiotic use in well-appearing term neonates born to mothers with chorioamnionitis. J Perinatol. 2017;37(12):1304-9. doi: 10.1038/jp.2017.137.

31. Kiser C, Nawab U, McKenna K, Aghai ZH. Role of guidelines on length of therapy in chorioamnionitis and neonatal sepsis. Pediatrics. 2014;133(6):992-8. doi: 10.1542/peds.2013-2927.

32. Mukhopadhyay S, Taylor JA, Von Kohorn I, Flaherman V, Burgos AE, Phillipi CA, et al. Variation in sepsis evaluation across a national network of nurseries. Pediatrics. 2017;139(3):e20162845. doi: 10.1542/peds.2016-2845.

33. Cantoni L, Ronfani L, Da Riol R, Demarini S. Perinatal Study Group of the Region Friuli-Venezia Giulia. Physical examination instead of laboratory tests for most infants born to mothers colonized with group B Streptococcus: support for the Centers for Disease Control and Prevention's 2010 recommendations. J Pediatr. 2013;163(2):568-73. doi: 10.1016/j.jpeds.2013.01.034

34. Kaiser Permanente Division of Research. Neonatal early-onset sepsis calculator. Available from: https:// neonatalsepsiscalculator.kaiserpermanente.org/. Accessed April 5; 2018.

35. Ayrapetyan M, Carola D, Lakshminrusimha S, BhandariV, Aghai
$\mathrm{ZH}$. Infants born to mothers with clinical chorioamnionitis: a cross-sectional survey on the use of early-onset sepsis risk calculator and prolonged use of antibiotics. Am J Perinatol. 2019;36(4):428-33. doi: 10.1055/s-0038-1668548.

36. Venkatesh KK, Jackon W, Hughes BL, Laughon MM, Thorp JM, Stamilio DM. Association of chorioamnionitis and its duration with neonatal morbidity and mortality. J Perinatol. 2019;39(5):673-82. doi: 10.1038/s41372-019-0322-0.

37. Committee on Obstetric Practice. Committee opinion no. 712: intrapartum management of intraamniotic infection. Obstet Gynecol. 2017;130(2):e95-e101. doi: 10.1097/ AOG.0000000000002236.

38. Afjeh SA, Sabzehei MK, Fahimzad SA, Shiva F, Shamshiri AR, Esmaili F. Antibiotic therapy for very low birth weight newborns in NICU. Iran J Pediatr. 2016;26(2):e2612. doi: 10.5812/ijp.2612.

39. Ramasethu J, Kawakita T. Antibiotic stewardship in perinatal and neonatal care. Semin Fetal Neonatal Med. 2017;22(5):27883. doi: 10.1016/j.siny.2017.07.001.

40. Greenland S, Mansournia MA, Altman DG. Sparse data bias: a problem hiding in plain sight. BMJ. 2016;352:i1981. doi: 10.1136/bmj.i1981. 